\title{
Acteurs locaux, gestion et développement des collectivités territoriales de Côte d'Ivoire : cas des communes de la région du Zanzan
}

\section{Adou Francois Kouadio}

\section{(2) OpenEdition Journals}

Édition électronique

URL : http://journals.openedition.org/cdg/2511

DOI : $10.4000 /$ cdg. 2511

ISSN : 2107-7266

Éditeur

UMR 245 - CESSMA

\section{Référence électronique}

Adou Francois Kouadio, «Acteurs locaux, gestion et développement des collectivités territoriales de Côte d'Ivoire : cas des communes de la région du Zanzan », Carnets de géographes [En ligne], 3 | 2011, mis en ligne le 01 décembre 2011, consulté le 23 septembre 2020. URL : http:// journals.openedition.org/cdg/2511; DOI : https://doi.org/10.4000/cdg.2511

Ce document a été généré automatiquement le 23 septembre 2020.

\section{cc) (†)}

La revue Carnets de géographes est mise à disposition selon les termes de la Licence Creative Commons Attribution - Pas d'Utilisation Commerciale - Pas de Modification 4.0 International. 


\title{
Acteurs locaux, gestion et développement des collectivités territoriales de Côte d'Ivoire : cas des communes de la région $\mathrm{du}$ Zanzan
}

\author{
Adou Francois Kouadio
}

1 Cette thèse interroge les liens entre gestion et développement des collectivités territoriales ivoiriennes par les acteurs locaux, à partir des communes de la région du Zanzan (nord-est de Côte d'Ivoire), région profondément rurale, pauvre et marginalisée. La crise politico-militaire du 19 septembre 2002 a entraîné une partition de la région: le nord administré par des chefs de guerre et le sud par des élus municipaux. Les principales questions posées dans ce travail sont les suivantes: comment ces acteurs locaux s'organisent-ils pour gérer et développer leurs territoires communaux? Le contexte de guerre a-t-il favorisé ou dilué l'autonomie des acteurs locaux? Quels sont les impacts socio-spatiaux de la gestion des communes?

2 L'étude de la gouvernance des communes du Zanzan, entre géographie sociale et géographie politique, s'inscrit dans une vaste réflexion sur la question de la décentralisation, en général et de la montée des acteurs locaux en Afrique, lien analysé sous l'angle de la démocratie participative, en particulier. Depuis les années 1980, la gouvernance des territoires communaux du Zanzan se présente comme un mécanisme participant de la politique de décentralisation. Il s'agit d'analyser les effets d'une politique impulsée par l'Etat sous la pression des bailleurs de fonds, une des premières d'Afrique subsaharienne. Peu de travaux ont mis en perspective la gestion des acteurs locaux dans un pays en guerre.

3 Sur le plan empirique, cette thèse met en lumière le rôle des différents élus, leur diversité et le jeu subtil des pouvoirs et des contre-pouvoirs, religieux, coutumiers, associatifs, de l'administration déconcentrée. L'état de guerre vécu au nord de la 
région, loin de permettre l'expression de la parole des citoyens, aboutit à la mise en place d'un régime militaire autoritaire, négateur de l'autonomie des élus locaux, à la différence des communes du sud de la région. Outre les acquis de la gestion municipale, notamment au niveau des services rendus et de la démocratie locale, cette thèse met aussi en évidence les limites nombreuses, qu'elles viennent de l'insuffisance des ressources, de l'absence de contrôle du foncier ou des luttes entre factions au niveau communal.

4 L'étude de la région du Zanzan constitue une expérience qui permet d'analyser le rôle et la stratégie des différents pouvoirs, les réactions des diverses populations, les conséquences du manque de moyens humains, juridiques et financiers et se pose, derrière les analyses, la question de la possibilité de décentraliser dans un pays en proie à une crise profonde et disposant surtout de ressources financières faibles.

\section{Méthodologie}

La méthodologie adoptée dans cette recherche est résolument qualitative, mêlant entretiens et observations. Elle s'appuie également sur une bibliographie. Plus précisément, il s'est agi de la compilation et de la synthèse de données provenant d'un important corpus bibliographique, issu de travaux historiques et anthropologiques en passant par des aspects généraux et théoriques des pouvoirs notamment le pouvoir politique.

Les communes enquêtées

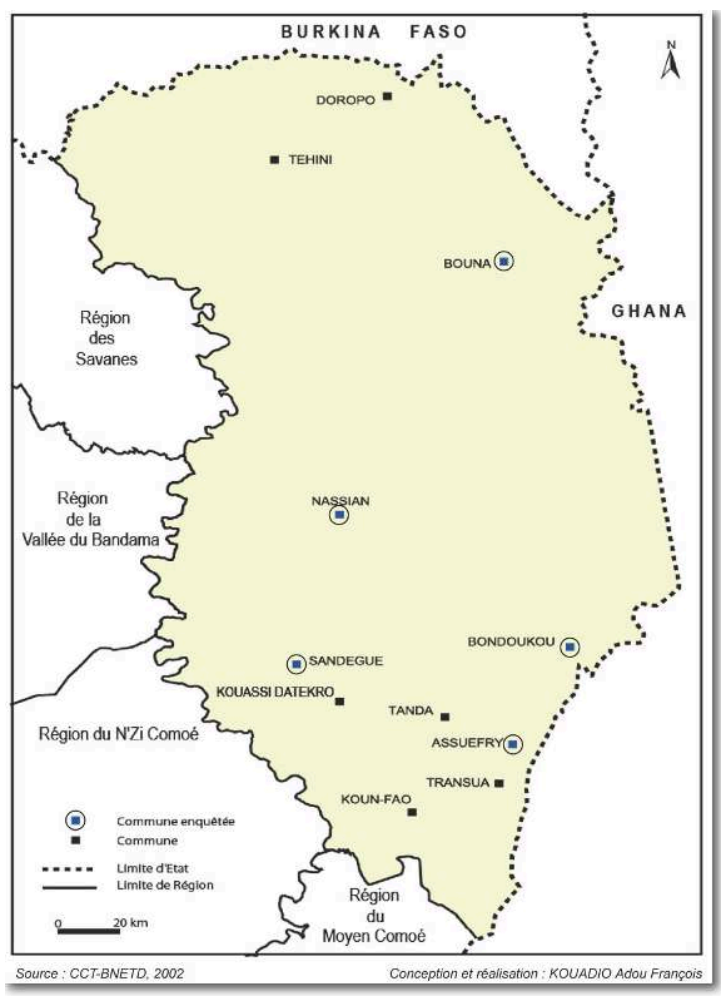

Source : CCT-BNETD ; conception et réalisation : KOUADIO Adou François 

empiriques plus larges et de favoriser de nouvelles pistes de recherche. Dans le sud de la région d'étude, en zone sous contrôle gouvernemental, j'ai opté pour une approche multi-située. Quatre communes (Assuefry, Bondoukou, Nassian et Sandégué) ont été choisies sur la base de la démographie, du statut administratif, de la localisation et de l'ancienneté. Trois groupes d'acteurs ont été soumis à des questionnaires. D'abord, les « acteurs d'en haut ", comprenant 3 administrateurs territoriaux, 7 maires et 4 adjoints aux maires, pour recueillir des éléments de leur gestion en termes de satisfactions et de problèmes liés à leur commune. Ensuite avec les personnes morales et communautaires, il s'est agi de voir comment les communautés s'organisent dans la marche de leur commune. Ce sont 3 chefs coutumiers, 3 religieux, 5 responsables socioéconomiques et 8 associations de jeunes, de femmes et de village. Enfin, les «acteurs d'en bas ", c'est-à-dire les habitants. C'est à cette échelle que le vécu quotidien de la population est perçu. Les populations étant les bénéficiaires des actions et des prestations des communes, leur rencontre s'impose en vue de cerner leurs attentes et leurs souhaits. L'autre objectif a été d'appréhender le vécu et la perception de la communalisation par les populations, mesurer le niveau et le degré de leur participation.

7 La méthode de choix raisonné m'a amené à prendre en compte dans l'élaboration de l'échantillon des critères comme l'ethnie, le sexe, l'âge, la profession, le niveau d'instruction et la dispersion géographique sur un effectif de 100 personnes en raison de 25 par commune.

Dans le nord de la région, seule la commune de Bouna a été enquêtée car elle fait office de centre principal des activités des hommes en armes. Ce sont 27 personnes dont deux soldats qui ont été enquêtées. Les entretiens ont porté sur les itinéraires individuels des combattants, des déterminants de leurs engagements respectifs, les formes de ponctions ou de taxes, la perception des populations sur la gestion de la commune.

\section{Fiche informative}

\author{
Lien électronique vers la thèse \\ http://www.theses.fr/2010PA010733

\section{Discipline \\ Géographie}

\section{Directeur} \\ Jean-Louis CHALEARD/ ATTA Koffi Lazare

\section{Université} \\ Université Paris 1 Panthéon Sorbonne
}

Membres du jury de thèse, soutenue le 7 février 2011

- Jean-Louis Chaléard, Professeur de Géographie à l'Université Paris 1 Panthéon Sorbonne, Directeur

- Michel Lesourd, Professeur de Géographie à l'Université de Rouen, Rapporteur - Yapi Diahou A., Professeur de Géographie à l'Université de Paris 8, Rapporteur et Président du jury 
- Atta Koffi, Maître de Conférences en Géographie à l'Université d'Abidjan Cocody, Co-Directeur

Situation professionnelle actuelle

Enseignant-chercheur, Université d'Abidjan Cocody

Courriel de l'auteur

adoufrancois[at]yahoo.fr

INDEX

Thèmes : Carnets de soutenances 